\title{
Classical Solutions of Path-Dependent PDEs and Functional Forward-Backward Stochastic Systems
}

\author{
Shaolin Ji ${ }^{1}$ and Shuzhen Yang ${ }^{2}$ \\ ${ }^{1}$ Institute for Financial Studies and Institute of Mathematics, Shandong University, Jinan, Shandong 250100, China \\ ${ }^{2}$ School of Mathematics, Shandong University, Jinan, Shandong 250100, China \\ Correspondence should be addressed to Shuzhen Yang; yangsz@mail.sdu.edu.cn
}

Received 20 February 2013; Accepted 20 April 2013

Academic Editor: Guangchen Wang

Copyright (c) 2013 S. Ji and S. Yang. This is an open access article distributed under the Creative Commons Attribution License, which permits unrestricted use, distribution, and reproduction in any medium, provided the original work is properly cited.

In this paper we study the relationship between functional forward-backward stochastic systems and path-dependent PDEs. In the framework of functional Itô calculus, we introduce a path-dependent PDE and prove that its solution is uniquely determined by a functional forward-backward stochastic system.

\section{Introduction}

It is well known that quasilinear parabolic partial differential equations are related to Markovian forward-backward stochastic differential equations (see [1-3]), which generalizes the classical Feynman-Kac formula. Recently in the frame-work of functional Itô calculus, a path-dependent PDE was introduced by Dupire [4] and the so-called functional Feynman-Kac formula was also obtained. For a recent account and development of this theory we refer the reader to [5-11].

In this paper, we study a functional forward-backward system and its relation to a quasilinear parabolic path-dependent PDE. In more details, the functional forward-backward system is described by the following forward-backward SDE:

$$
\begin{aligned}
X^{\gamma_{t}}(s)=\gamma_{t}(t)+\int_{t}^{s} b\left(X_{r}^{\gamma_{t}}\right) d r+\int_{t}^{s} \sigma\left(X_{r}^{\gamma_{t}}\right) d W(r), \\
X^{\gamma_{t}}(r)=\gamma_{t}(r), \quad 0 \leq r \leq t, \\
Y^{\gamma_{t}}(s)=g\left(X_{T}^{\gamma_{t}}\right)-\int_{s}^{T} h\left(X_{r}^{\gamma_{t}}, Y^{\gamma_{t}}(r), Z^{\gamma_{t}}(r)\right) d r \\
\quad-\int_{s}^{T} Z^{\gamma_{t}}(r) d W(r), \quad s \in[t, T] .
\end{aligned}
$$

Equation (1) is an uncoupled functional forward-backward system, its general the results of [8], and there are many applications of the uncoupled functional forward-backward system in optimal control problem. The main difference is that we give a weaker requirement of $g$ and $h$ about $X$, and we also establish some estimates and regularity results for the solution with respect to paths. Then, we prove that the solution of (1) is the unique classical solution of the following path-dependent PDE:

$$
\begin{aligned}
D_{t} u\left(\gamma_{t}\right)+\mathscr{L} u\left(\gamma_{t}\right) & =h\left(\gamma_{t}, u\left(\gamma_{t}\right), D_{x} u\left(\gamma_{t}\right) \sigma\left(\gamma_{t}\right)\right), \\
u\left(\gamma_{T}\right) & =g\left(\gamma_{T}\right), \quad \gamma_{T} \in \Lambda,
\end{aligned}
$$

where

$$
\mathscr{L} u=\frac{1}{2} \operatorname{tr}\left[\sigma \sigma^{T} D_{x x} u\right]+\left\langle b, D_{x} u\right\rangle
$$

The paper is organized as follows: in Section 2, we give the notations and results on functional FBSDEs and functional Itô calculus. Some estimates and regularity results for the solution of FBSDEs are established in Section 3. Finally, we prove the relationship between functional FBSDEs and pathdependent PDEs in Section 4. 


\section{Preliminaries}

2.1. Functional FBSDEs. Let $\Omega=C\left([0, T] ; \mathbb{R}^{d}\right)$ and let $P$ be the Wiener measure on $(\Omega, \mathbb{B}(\Omega))$. We denote by $W=$ $\left(W(t)_{t \in[0, T]}\right)$ the cannonical Wiener process, with $W(t, \omega)=$ $\omega(t), t \in[0, T], \omega \in \Omega$. For any $t \in[0, T]$ we denote by $\mathscr{F}_{t}$ the $P$-completion of $\sigma(W(s), s \in[0, t])$.

For any $t \in[0, T]$, we denote by $L^{2}\left(\Omega, \mathscr{F}_{t} ; \mathbb{R}^{n}\right)$ the set of all square integrable $\mathscr{F}_{t}$-measurable random variables, $M^{2}\left(0, T ; \mathbb{R}^{n}\right)$ the set of all $\mathbb{R}^{n}$-valued $\mathscr{F}_{t}$-adapted processes $\vartheta(\cdot)$ such that

$$
E \int_{0}^{T}|\vartheta(s)|^{2} d s<+\infty .
$$

Let $t \in[0, T]$ and $\gamma_{t} \in \Lambda$. For every $s \in[t, T]$, we consider the following functional forward-backward SDEs:

$$
\begin{gathered}
X^{\gamma_{t}}(s)=\gamma_{t}(t)+\int_{t}^{s} b\left(X_{r}^{\gamma_{t}}\right) d r+\int_{t}^{s} \sigma\left(X_{r}^{\gamma_{t}}\right) d W(r), \\
Y^{\gamma_{t}}(s)=g\left(X_{T}^{\gamma_{t}}\right)-\int_{s}^{T} h\left(X_{r}^{\gamma_{t}}, Y^{\gamma_{t}}(r), Z^{\gamma_{t}}(r)\right) d r \\
-\int_{s}^{T} Z^{\gamma_{t}}(r) d W(r),
\end{gathered}
$$

where

$$
X^{\gamma_{t}}(s)=\gamma_{t}(s), \quad s \in[0, t]
$$

The processes $X, Y, Z$ take values in $\mathbb{R}^{n}, \mathbb{R}^{n}, \mathbb{R}^{n \times d} ; b, h, \sigma$, and $g$ take values in $\mathbb{R}^{n}, \mathbb{R}^{n}, \mathbb{R}^{n \times d}$, and $\mathbb{R}^{n}$. Equations (5) and (6) can be rewritten as

$$
\begin{aligned}
& d X^{\gamma_{t}}(s)=b\left(X_{s}^{\gamma_{t}}\right) d s+\sigma\left(X_{s}^{\gamma_{t}}\right) d W(s), \\
& d Y^{\gamma_{t}}(s)=h\left(X_{s}^{\gamma_{t}}, Y^{\gamma_{t}}(s), Z^{\gamma_{t}}(s)\right) d s+Z^{\gamma_{t}}(s) d W(s), \\
& X^{\gamma_{t}}(t)=\gamma_{t}(t), \quad Y^{\gamma_{t}}(T)=g\left(X_{T}^{\gamma_{t}}\right) .
\end{aligned}
$$

For $z \in \mathbb{R}^{n \times d}$, we define $|z|=\left\{\operatorname{tr}\left(z z^{T}\right)\right\}^{1 / 2}$. For $z^{1} \in \mathbb{R}^{n \times d}$, $z^{2} \in \mathbb{R}^{n \times d}$,

$$
\left(\left(z^{1}, z^{2}\right)\right)=\operatorname{tr}\left(z^{1}\left(z^{2}\right)^{T}\right)
$$

and for $u^{1}=\left(y^{1}, z^{1}\right) \in \mathbb{R}^{n} \times \mathbb{R}^{n \times d}, u^{2}=\left(y^{2}, z^{2}\right) \in \mathbb{R}^{n} \times \mathbb{R}^{n \times d}$

$$
\left[u^{1}, u^{2}\right]=\left\langle y^{1}, y^{2}\right\rangle+\left(\left(z^{1}, z^{2}\right)\right) .
$$

We give the following assumption.

Assumption 1. For all $x_{T}^{1}, x_{T}^{2} \in \Lambda, b\left(x^{1}\right), \sigma\left(x^{1}\right) \in M^{2}$, and $t \in[0, T]$, there exists a constant $c_{1}>0$, such that

$$
\left|b\left(x_{t}^{1}\right)-b\left(x_{t}^{2}\right)\right|+\left|\sigma\left(x_{t}^{1}\right)-\sigma\left(x_{t}^{2}\right)\right| \leq c_{1}\left\|x_{t}^{1}-x_{t}^{2}\right\|, \quad \text { a.e. }
$$

and for all $x_{t} \in \Lambda$,

$$
\left|b\left(x_{t}\right)\right|+\left|\sigma\left(x_{t}\right)\right| \leq c_{1}\left(1+\left\|x_{t}\right\|\right), \quad \text { a.e. }
$$

Definition 2. $X:[0, T] \times \Omega \rightarrow \mathbb{R}^{n}$ is called an adapted solution of (5), if $X \in M^{2}\left(0, T ; \mathbb{R}^{n}\right)$, and it satisfies (5) $P$-a.s.

Then we have the following theorem (see [12]).

Theorem 3. Let Assumption 1 hold, then there exists a unique adapted solution $X$ for (5).

2.2. Functional Itô Calculus. The following notations and tools are mainly from Dupire [4]. Let $T>0$ be fixed. For each $t \in[0, T]$, we denote by $\Lambda_{t}$ the set of càdlàg $\mathbb{R}^{d}$-valued functions on $[0, t]$. For each $\gamma \in \Lambda_{T}$ the value of $\gamma$ at time $s \in$ $[0, T]$ is denoted by $\gamma(s)$. Thus $\gamma=\gamma(s)_{0 \leq s \leq T}$ is a càdlàg process on $[0, T]$ and its value at time $s$ is $\gamma(s)$. The path of $\gamma$ up to time $t$ is denoted by $\gamma_{t}$, that is, $\gamma_{t}=\gamma(s)_{0 \leq s \leq t} \in \Lambda_{t}$. We denote $\Lambda=\bigcup_{t \in[0, T]} \Lambda_{t}$. For each $\gamma_{t} \in \Lambda$ and $x \in \mathbb{R}^{d}$ we denote by $\gamma_{t}(s)$ the value of $\gamma_{t}$ at $s \in[0, t]$ and $\gamma_{t}^{x}:=\left(\gamma_{t}(s)_{0 \leq s<t}, \gamma_{t}(t)+x\right)$ which is also an element in $\Lambda_{t}$.

Let $\langle\cdot, \cdot\rangle$ and $|\cdot|$ denote the inner product and norm in $\mathbb{R}^{n}$. We now define a distance on $\Lambda$. For each $0 \leq t, \bar{t} \leq T$ and $\gamma_{t}, \bar{\gamma}_{\bar{t}} \in \Lambda$, we denote

$$
\begin{aligned}
& \left\|\gamma_{t}\right\|:=\sup _{s \in[0, t]}\left|\gamma_{t}(s)\right|, \\
& \left\|\gamma_{t}-\bar{\gamma}_{\bar{t}}\right\|:=\sup _{s \in[0, t \vee \bar{t}]}\left|\gamma_{t}(s \wedge t)-\bar{\gamma}_{\bar{t}}(s \wedge \bar{t})\right|, \\
& d_{\infty}\left(\gamma_{t}, \bar{\gamma}_{\bar{t}}\right):=\sup _{0 \leq s \leq t \vee \bar{t}}\left|\gamma_{t}(s \wedge t)-\bar{\gamma}_{\bar{t}}(s \wedge \bar{t})\right|+|t-\bar{t}| .
\end{aligned}
$$

It is obvious that $\Lambda_{t}$ is a Banach space with respect to $\|\cdot\|$ and $d_{\infty}$ is not a norm.

Definition 4. A function $u: \Lambda \mapsto \mathbb{R}$ is said to be $\Lambda$ continuous at $\gamma_{t} \in \Lambda$, if for any $\varepsilon>0$ there exists $\delta>0$ such that for each $\bar{\gamma}_{\bar{t}} \in \Lambda$ with $d_{\infty}\left(\gamma_{t}, \bar{\gamma}_{\bar{t}}\right)<\delta$, we have $\left|u\left(\gamma_{t}\right)-u\left(\bar{\gamma}_{\bar{t}}\right)\right|<\varepsilon . u$ is said to be $\Lambda$-continuous if it is $\Lambda$ continuous at each $\gamma_{t} \in \Lambda$.

Definition 5. Let $u: \Lambda \mapsto \mathbb{R}$ and $\gamma_{t} \in \Lambda$ be given. If there exists $p \in \mathbb{R}^{d}$, such that

$$
u\left(\gamma_{t}^{x}\right)=u\left(\gamma_{t}\right)+\langle p, x\rangle+o(|x|) \text { as } x \longrightarrow 0, x \in \mathbb{R}^{d},
$$

then we say that $u$ is vertically differentiable at $\gamma_{t}$ and denote the gradient of $D_{x} u\left(\gamma_{t}\right)=p$. If $D_{x} u\left(\gamma_{t}\right)$ exists for each $\gamma_{t} \in \Lambda$, $u$ is said to be vertically differentiable in $\Lambda$.

We can similarly define the Hessian $D_{x x} u\left(\gamma_{t}\right)$. It is an $\mathbb{S}(d)$-valued function defined on $\Lambda$, where $\mathbb{S}(d)$ is the space of all $d \times d$ symmetric matrices.

For each $\gamma_{t} \in \Lambda$ we denote

$$
\gamma_{t, s}(r)=\gamma_{t}(r) \mathbf{1}_{[0, t)}(r)+\gamma_{t}(t) \mathbf{1}_{[t, s]}(r), \quad r \in[0, s] .
$$

It is clear that $\gamma_{\mathrm{t}, \mathrm{s}} \in \Lambda_{\mathrm{s}}$.

Definition 6. For a given $\gamma_{t} \in \Lambda$ if we have

$$
u\left(\gamma_{t, s}\right)=u\left(\gamma_{t}\right)+a(s-t)+o(|s-t|) \quad \text { as } s \longrightarrow t, s \geq t
$$


then we say that $u\left(\gamma_{t}\right)$ is (horizontally) differentiable in $t$ at $\gamma_{t}$ and $D_{t} u\left(\gamma_{t}\right)=a . u$ is said to be horizontally differentiable in $\Lambda$ if $D_{t} u\left(\gamma_{t}\right)$ exists for each $\gamma_{t} \in \Lambda$.

Definition 7. Define $\mathbb{C}^{j, k}(\Lambda)$ as the set of function $u:=$ $\left(u\left(\gamma_{t}\right)\right)_{\gamma_{t} \in \Lambda}$ defined on $\Lambda$ which are $j$ times horizontally and $k$ times vertically differentiable in $\Lambda$ such that all these derivatives are $\Lambda$-continuous.

The following Itô formula was firstly obtained by Dupire [4] and then generalized by Cont and Fournié [5-7].

Theorem 8 (functional Itô's formula). Let $\left(\Omega, \mathscr{F}_{,}\left(\mathscr{F}_{t}\right)_{t \in[0, T]}\right.$, $P)$ be a probability space, if $X$ is a continuous semi-martingale and $u$ is in $\mathbb{C}^{1,2}(\Lambda)$, then for any $t \in[0, T)$,

$$
\begin{aligned}
u\left(X_{t}\right)-u\left(X_{0}\right)= & \int_{0}^{t} D_{s} u\left(X_{s}\right) d s+\int_{0}^{t} D_{x} u\left(X_{s}\right) d X(s) \\
& +\frac{1}{2} \int_{0}^{t} D_{x x} u\left(X_{s}\right) d\langle X\rangle(s), \quad P \text {-a.s. }
\end{aligned}
$$

\section{Regularity}

We first recall some notions in Pardoux and Peng [2]. $C^{n}\left(\mathbb{R}^{p} ; \mathbb{R}^{q}\right), C_{b}^{n}\left(\mathbb{R}^{p} ; \mathbb{R}^{q}\right), C_{p}^{n}\left(\mathbb{R}^{p} ; \mathbb{R}^{q}\right)$ will denote, respectively, the set of functions of class $C^{n}$ from $\mathbb{R}^{p}$ into $\mathbb{R}^{q}$, the set of those functions of class $C_{b}^{n}$ whose partial derivatives of order less than or equal to $n$ are bounded, and the set of those functions of class $C_{p}^{n}$ which, together with all their partial derivatives of order less than or equal to $n$, grow at most like a polynomial function of the variable $x$ at infinity.

Now we give the definition of derivatives in our context. Under Assumption 1 we have that

$$
\begin{gathered}
d X^{\gamma_{t}}(s)=b\left(X_{s}^{\gamma_{t}}\right) d s+\sigma\left(X_{s}^{\gamma_{t}}\right) d W(s), \\
X^{\gamma_{t}}(t)=\gamma_{t}(t),
\end{gathered}
$$

has a unique solution. For $t \leq s \leq T$, set

$$
\begin{gathered}
\widetilde{\Lambda}_{\gamma_{t}, s}:=\left\{\bar{\gamma}_{s}: \bar{\gamma}(h)=X^{\gamma_{t}}(h, \omega), 0 \leq h \leq s, \omega \in \Omega\right\}, \\
\widetilde{\Lambda}_{t, s}:=\bigcup_{\gamma_{t} \in \Lambda_{t}} \widetilde{\Lambda}_{\gamma_{t}, s}, \quad \widetilde{\Lambda}_{t}:=\bigcup_{t \leq s \leq T} \widetilde{\Lambda}_{t, s} .
\end{gathered}
$$

Then the following definition of derivatives will be used frequently in the sequel.

Definition 9. An $\mathbb{R}^{n}$-valued function $g$ is said to be in $C^{2}\left(\widetilde{\Lambda}_{\gamma_{t}, T}\right)$, if for $\gamma_{1} \in \widetilde{\Lambda}_{\gamma_{t}, T}$ and $\gamma_{2} \in \widetilde{\Lambda}_{\gamma_{t}^{y}, T}$, there exist $p_{1} \in \mathbb{R}^{d}$ and $p_{2} \in \mathcal{S}^{d}$ ( $\mathcal{S}^{d}$ is the set of all $d$ order symmetric matrix) such that

$$
g\left(\gamma_{2}\right)-g\left(\gamma_{1}\right)=\left\langle p_{1}, y\right\rangle+\frac{1}{2}\left\langle p_{2} y, y\right\rangle+o\left(|y|^{2}\right), \quad x \in \mathbb{R}^{d} .
$$

We denote $g_{\gamma_{t}}^{\prime}\left(\gamma_{1}\right):=p_{1}$, and $g_{\gamma_{t}}^{\prime \prime}\left(\gamma_{1}\right):=p_{2} \cdot g$ is said to be in $C_{l, l i p}^{2}\left(\widetilde{\Lambda}_{t, T}\right)$ if $g_{\gamma_{t}}^{\prime}(\gamma)$ and $g_{\gamma_{t}}^{\prime \prime}(\gamma)$ exist for each $\gamma_{t} \in \Lambda_{t}$, and there exist some constants $C \geq 0$ and $k \geq 0$ depending only on $g$ such that for each $\gamma, \bar{\gamma} \in \Lambda_{T}, t, s \in[0, T]$,

$$
|g(\gamma)-g(\bar{\gamma})| \leq C\left(\|\gamma\|^{k}+\|\bar{\gamma}\|^{k}\right)\|\gamma-\bar{\gamma}\|
$$

and for each $\gamma \in \widetilde{\Lambda}_{t, T}, \bar{\gamma} \in \widetilde{\Lambda}_{s, T}, t, s \in[0, T]$,

$$
\left|\Phi_{\gamma_{t}}(\gamma)-\Phi_{\gamma_{s}}(\bar{\gamma})\right| \leq C\left(\|\gamma\|^{k}+\|\bar{\gamma}\|^{k}\right)(|t-s|+\|\gamma-\bar{\gamma}\|)
$$

with $\Phi=g_{\gamma_{t}}^{\prime}(\gamma), g_{\gamma_{t}}^{\prime \prime}(\gamma)$. We can also define $C^{2}\left(\widetilde{\Lambda}_{t, s}\right)$, $C_{l, l i p}^{2}\left(\widetilde{\Lambda}_{t, s}\right), C_{l, l i p}^{1}\left(\widetilde{\Lambda}_{t, s}\right), C_{l, l i p}\left(\widetilde{\Lambda}_{t, s}\right)$ and $C^{2}\left(\widetilde{\Lambda}_{t}\right), C_{l, l i p}^{2}\left(\widetilde{\Lambda}_{t}\right)$, $C_{l, l i p}^{1}\left(\widetilde{\Lambda}_{t}\right), C_{l, l i p}\left(\widetilde{\Lambda}_{t}\right)$.

Now we consider the solvability of (6).

Assumption 10. Let $g$ be an $\mathbb{R}^{n}$-valued function on $\Lambda_{T}$. Moreover $g \in C_{l, l i p}^{2}\left(\widetilde{\Lambda}_{t, T}\right)$ with the Lipschitz constants $C$ and $k$.

Assumption 11. Let $h\left(\gamma_{t}, y, z\right)=\bar{h}(t, \gamma(t), y, z)$, where $\bar{h}$ : $[0, T] \times \mathbb{R}^{n} \times \mathbb{R}^{n} \times \mathbb{R}^{n \times d} \mapsto \mathbb{R}^{n}$ is such that $(t, r, y, z) \mapsto$ $\bar{\Psi}(t, r, y, z)$ is of class $C_{p}^{0,3}\left([0, T] \times \mathbb{R}^{n} \times \mathbb{R}^{n} \times \mathbb{R}^{n \times d} ; \mathbb{R}^{n}\right)$ and the first order partial derivatives in $r, y$, and $z$ are bounded, as well as their derivatives of up to order two with respect to $y, z$.

It is obvious under Assumptions 1, 10, and 11 the FBSDE (5) and (6) has a unique solution (see [12-14]).

3.1. Regularity of the Solution of FBSDEs. We assume the Lipschitz constants with respect to $b, \sigma, h$ are $C$ and $k$. Then we have the following estimates for the solutions of FBSDE (5) and (6).

Lemma 12. Under Assumptions 1, 10, and 11, for all $p \geq 2$ there exist $C_{2}$ and $q$ depending only on $C, T, k, x$ such that

$$
\begin{gathered}
E\left[\sup _{s \in[t, T]}\left|X^{\gamma_{t}}(s)\right|^{p}\right] \leq C_{2}\left(1+\left\|\gamma_{t}\right\|^{p}\right), \\
E\left[\sup _{s \in[t, T]}\left|Y^{\gamma_{t}}(s)\right|^{p}\right] \leq C_{2}\left(1+\left\|\gamma_{t}\right\|^{q}\right), \\
E\left[\left(\int_{t}^{T}\left|Z^{\gamma_{t}}(s)\right|^{2} d s\right)^{p / 2}\right] \leq C_{2}\left(1+\left\|\gamma_{t}\right\|^{q}\right) .
\end{gathered}
$$

Proof. To simplify presentation, we only study the case $n=$ $d=1$, and $p=2$. 

that

The application of Itô's formula to $\left(Y_{\gamma_{t}, x}(s)\right)^{2} e^{\beta_{1} s}$ yields

$$
\begin{aligned}
& \left(Y^{\gamma_{t},}(s)\right)^{2} e^{\beta_{1} s}+\int_{s}^{T} e^{\beta_{1} r}\left[\left(Z^{\gamma_{t}}(r)\right)^{2}+\beta_{1}\left(Y^{\gamma_{t}}(r)\right)^{2}\right] d r \\
& =g^{2}\left(X_{T}^{\gamma_{t}}\right) e^{\beta_{1} T} \\
& \quad-\int_{s}^{T} 2 e^{\beta_{1} r} Y^{\gamma_{t}}(r) h\left(X_{r}^{\gamma_{t}}, Y^{\gamma_{t}}(r), Z^{\gamma_{t}}(r)\right) d r \\
& \quad-\int_{s}^{T} 2 e^{\beta_{1} r} Y^{\gamma_{t}}(r) Z^{\gamma_{t}}(r) d W(r) .
\end{aligned}
$$

So

$$
\begin{gathered}
\left(Y^{\gamma_{t}}(s)\right)^{2}+E\left[\int _ { s } ^ { T } e ^ { \beta _ { 1 } ( r - s ) } \left[\left(Z^{\gamma_{t}}(r)\right)^{2}\right.\right. \\
\left.\left.+\beta_{1}\left(Y^{\gamma_{t}}(r)\right)^{2}\right] d r \mid \mathscr{F}_{s}\right] \\
=E\left[g^{2}\left(X_{T}^{\gamma_{t}}\right) e^{\beta_{1}(T-s)} \mid \mathscr{F}_{s}\right] \\
-E\left[\int_{s}^{T} 2 e^{\beta_{1}(r-s)} Y^{\gamma_{t}}(r)\right. \\
\left.\quad \times h\left(X_{r}^{\gamma_{t}}, Y^{\gamma_{t}}(r), Z^{\gamma_{t}}(r)\right) d r \mid \mathscr{F}_{s}\right] .
\end{gathered}
$$

Then we have

$$
\begin{aligned}
& E \sup _{t \leq s \leq T}\left(Y^{\gamma_{t}}(s)\right)^{2} \\
&+ E\left[\int_{t}^{T} e^{\beta_{1}(r-t)}\left[\left(Z^{\gamma_{t}}(r)\right)^{2}+\beta_{1}\left(Y^{\gamma_{t}}(T)\right)^{2}\right] d r\right] \\
& \leq E\left[g^{2}\left(X_{T}^{\gamma_{t}}\right) e^{\beta_{1}(T-t)}\right] \\
&+ E\left[\int_{t}^{T} e^{\beta_{1}(r-t)} \frac{2}{\beta_{1}} h^{2}\left(X_{r}^{\gamma_{t}}, Y^{\gamma_{t}}(r), Z^{\gamma_{t}}(r)\right) d r\right] \\
&+ E\left[\int_{t}^{T} e^{\beta_{1}(r-t)} \frac{\beta_{1}}{2}\left(Y^{\gamma_{t}}(r)\right)^{2} d(r)\right], \\
& E \sup _{t \leq s \leq T}\left(Y^{\gamma_{t}}(s)\right)^{2} \\
&+E\left[\int_{t}^{T} e^{\beta_{1}(r-t)}\left[\left(Z^{\gamma_{t}}(r)\right)^{2}+\frac{\beta_{1}}{2}\left(Y^{\gamma_{t}}(r)\right)^{2}\right] d r\right] \\
& \leq E\left[g^{2}\left(X_{T}^{\gamma_{t}}\right) e^{\beta_{1}(T-t)}\right] \\
&+E\left[\int_{t}^{T} e^{\beta_{1}(r-t)} \frac{2}{\beta_{1}} h^{2}\left(X_{r}^{\gamma_{t}}, Y^{\gamma_{t}}(r), Z^{\gamma_{t}}(r)\right) d r\right] .
\end{aligned}
$$

Applying Itô's formula to $\left(X^{\gamma_{t}, x}(s)\right)^{2}$ yields that

$$
\begin{aligned}
\left(X^{\gamma_{t}}(s)\right)^{2}= & \gamma_{t}(t)^{2}+\int_{t}^{s} 2 X^{\gamma_{t}}(r) b\left(X_{r}^{\gamma_{t}}\right) d r \\
& +\int_{t}^{s} 2 X^{\gamma_{t}}(r) \sigma\left(X_{r}^{\gamma_{t}}\right) d W(r)+\int_{t}^{s} \sigma^{2}\left(X_{r}^{\gamma_{t}}\right) d r .
\end{aligned}
$$

By inequality $2 a b \leq a^{2}+b^{2}$ and Burkholder-Davis-Gundy's inequality, there is a $C_{0}$ such that

$$
\begin{aligned}
& E \sup _{t \leq r \leq s}\left(X^{\gamma_{t}}(r)\right)^{2} \\
& \leq C_{0}\left[\gamma_{t}(t)^{2}+E \int_{t}^{s} b^{2}\left(X_{r}^{\gamma_{t}}\right) d r\right. \\
&\left.\quad+E \int_{t}^{s}\left(X^{\gamma_{t}}(r)\right)^{2} d r+E \int_{t}^{s} \sigma^{2}\left(X_{r}^{\gamma_{t}}\right) d r\right] .
\end{aligned}
$$

By Assumption 1 and Gronwall's inequality, from (29) we have (note that $C_{0}$ will change line by line)

$$
E \sup _{t \leq r \leq T}\left(X^{\gamma_{t}}(r)\right)^{2} \leq C_{0}\left(1+\left\|\gamma_{t}\right\|^{2}\right) .
$$

By Assumptions 10 and 11 and taking $\beta_{1}=4 C^{2}+1$, from (27) we have

$$
\begin{aligned}
E \sup _{t \leq s \leq T}\left(Y^{\gamma_{t}}(s)\right)^{2} & \\
& +E\left[\int_{t}^{T}\left[\left(Z^{\gamma_{t}}(r)\right)^{2}+\left(Y^{\gamma_{t}}(r)\right)^{2}\right] d r\right] \\
& \leq C_{0}\left(1+P \gamma_{t} P^{q}\right),
\end{aligned}
$$

where $q=2(1+k)$. Similarly we can get the same result for $p \geq 2$.

This completes the proof.

Now we study the regularity properties of the solution of FBSDE (5), (6) with respect to the "parameter" $\gamma_{t}$. For $0 \leq s<$ $t \leq T$, define $Y^{\gamma_{t}}(s)=Y^{\gamma_{t}}(s \vee t)$ and $Z^{\gamma_{t}}(s)=0$.

Theorem 13. Under Assumptions 1, 10 and 11, for all $p \geq 2$ there exist $C_{2}$ and $q$ depending only on $C, c_{2}, x$ such that for any $t, \bar{t} \in[0, T], \gamma_{t}, \bar{\gamma}_{\bar{t}}$, and $h, \bar{h} \in \mathbb{R} \backslash\{0\}$

(i)

$$
\begin{aligned}
& E\left[\sup _{u \in[t \overline{\bar{t}} T]}\left|Y^{\gamma_{t}}(u)-Y^{\bar{\gamma}_{\bar{t}}}(u)\right|^{p}\right] \\
& \quad \leq C_{2}\left(1+\left\|\gamma_{t}\right\|^{q}+\left\|\bar{\gamma}_{\bar{t}}\right\|^{q}\right)\left(\left\|\gamma_{t}-\bar{\gamma}_{\bar{t}}\right\|^{p}+|t-\bar{t}|^{p / 2}\right),
\end{aligned}
$$

(ii)

$$
\begin{aligned}
& E\left[\left|\int_{t \vee \bar{t}}^{T}\right| Z^{\gamma_{t}}(u)-\left.\left.Z^{\overline{\gamma_{\bar{t}}}}(u)\right|^{2} d u\right|^{p / 2}\right] \\
& \quad \leq C_{2}\left(1+\left\|\gamma_{t}\right\|^{q}+\left\|\bar{\gamma}_{\bar{t}}\right\|^{q}\right)\left(\left\|\gamma_{t}-\bar{\gamma}_{\bar{t}}\right\|^{p}+|t-\bar{t}|^{p / 2}\right),
\end{aligned}
$$


Mathematical Problems in Engineering

5

(iii)

$$
\begin{gathered}
E\left[\sup _{u \in[t \overline{\bar{t}}, T]}\left|\Delta_{h}^{i} Y^{\gamma_{t}}(u)-\Delta_{h}^{i} Y^{\bar{\gamma}_{\bar{t}}}(u)\right|^{p}\right] \\
\leq C_{2}\left(1+\left\|\gamma_{t}\right\|^{q}+\left\|\bar{\gamma}_{\bar{t}}\right\|^{q}+|h|^{q}+|\bar{h}|^{q}\right) \\
\quad \times\left(|h-\bar{h}|^{p}+\left\|\gamma_{t}-\bar{\gamma}_{\bar{t}}\right\|^{p}+|t-\bar{t}|^{p / 2}\right),
\end{gathered}
$$

(iv)

$$
\begin{gathered}
E\left[\left|\int_{t \vee \bar{t}}^{T}\right| \Delta_{h}^{i} Z^{\gamma_{t}}(u)-\left.\left.\Delta_{h}^{i} Z^{\bar{\gamma}_{\bar{t}}}(u)\right|^{2} d u\right|^{p / 2}\right] \\
\leq C_{2}\left(1+\left\|\gamma_{t}\right\|^{q}+\left\|\bar{\gamma}_{\bar{t}}\right\|^{q}+|h|^{q}+|\bar{h}|^{q}\right) \\
\quad \times\left(|h-\bar{h}|^{p}+\left\|\gamma_{t}-\bar{\gamma}_{\bar{t}}\right\|^{p}+|t-\bar{t}|^{p / 2}\right),
\end{gathered}
$$

where

$$
\begin{aligned}
\Delta_{h}^{i} Y^{\gamma_{t}, x}(s) & =\frac{1}{h}\left(Y^{\gamma_{t}^{h_{e_{i}}}}(s)-Y^{\gamma_{t}}(s)\right), \\
\Delta_{h}^{i} Z^{\gamma_{t}}(s) & =\frac{1}{h}\left(Z^{\gamma_{t}^{h_{e_{i}}}}(s)-Z^{\gamma_{t}}(s)\right)
\end{aligned}
$$

and $\left(e_{1}, \ldots, e_{n}\right)$ is an orthonormal basis of $\mathbb{R}^{n}$.

Proof. $\left(Y^{\gamma_{t}}-Y^{\bar{\gamma}_{\bar{t}}}, Z^{\gamma_{t}}-Z^{\bar{\gamma}_{\bar{t}}}\right)$ can be formed as a linearized BSDE: for each $s \in[t \vee \bar{t}, T]$,

$$
\begin{aligned}
Y^{\gamma_{t}}(s)-Y^{\bar{\gamma}_{\bar{t}}}(s) & \\
= & g\left(X_{T}^{\gamma_{t}}\right)-g\left(X_{T}^{\bar{\gamma}_{\bar{t}}}\right) \\
& +\int_{s}^{T}\left[h\left(X_{r}^{\gamma_{t}}, Y^{\gamma_{t}}(r), Z^{\gamma_{t}}(r)\right)\right. \\
& \left.+h\left(X_{r}^{\bar{\gamma}_{\bar{t}}}, Y^{\bar{\gamma}_{\bar{t}}}(r), Z^{\bar{\gamma}_{\bar{t}}}(r)\right)\right] d r \\
= & g\left(X_{T}^{T}\left(Z^{\gamma_{t}}\right)-g(r)-Z^{\bar{\gamma}_{\bar{t}}}(r)\right) d W\left(X_{T}^{\bar{\gamma}_{\bar{t}}},\right) \\
& -\int_{s}^{T}\left[\widehat{\alpha}_{\gamma_{t}, \bar{\gamma}_{\bar{t}}}(r)+\widehat{\beta}_{\gamma_{t}, \bar{\gamma}_{\bar{t}}}\left(Y^{\gamma_{t}}(r)-Y^{\bar{\gamma}_{\bar{t}}}(r)\right)\right. \\
& \left.+\widehat{\delta}_{\gamma_{t}, \bar{\gamma}_{\bar{t}}}\left(Z^{\gamma_{t}}(r)-Z^{\bar{\gamma}_{\bar{t}}}(r)\right)\right] d r \\
& +\int_{s}^{T}\left(Z^{\gamma_{t}}(r)-Z^{\bar{\gamma}_{\bar{t}}}(r)\right) d W(r),
\end{aligned}
$$

where (with $\left.U^{\gamma_{t}}=\left(Y^{\gamma_{t}}, Z^{\gamma_{t}}\right)\right)$

$$
\begin{aligned}
& \widehat{\alpha}_{\gamma_{t}, \bar{\gamma}_{\bar{t}}}(r)=h\left(X_{r}^{\gamma_{t}}, Y^{\bar{\gamma}_{\bar{t}}}(r), Z^{\bar{\gamma}_{\bar{t}}}(r)\right)-h\left(X_{r}^{\bar{\gamma}_{\bar{t}}}, Y^{\bar{\gamma}_{\bar{t}}}(r), Z^{\bar{\gamma}_{\bar{t}}}(r)\right), \\
& \widehat{\beta}_{\gamma_{t}, \bar{\gamma}_{\bar{t}}}(r)=\int_{0}^{1} \frac{\partial h}{\partial y}\left(X_{r}^{\gamma_{t}}, U^{\bar{\gamma}_{\bar{t}}}(r)+\theta\left(U^{\gamma_{t}}(r)-U^{\bar{\gamma}_{\bar{t}}}(r)\right)\right) d \theta, \\
& \widehat{\delta}_{\gamma_{t}, \bar{\gamma}_{\bar{t}}}(r)=\int_{0}^{1} \frac{\partial h}{\partial z}\left(X_{r}^{\gamma_{t}}, U^{\bar{\gamma}_{\bar{t}}}(r)+\theta\left(U^{\gamma_{t}}(r)-U^{\bar{\gamma}_{\bar{t}}}(r)\right)\right) d \theta .
\end{aligned}
$$

Under Assumptions 10, 11, using the same method as in Lemma 12, we get the first three inequalities.

For the next three inequalities, we write $\left(\Delta_{h}^{i} Y^{\gamma_{t}}, \Delta_{h}^{i} Z^{\gamma_{t}}\right)$ as the solution of the following linearized BSDE:

$$
\begin{aligned}
& \Delta_{h}^{i} Y^{\gamma_{t}}(s) \\
& =\frac{1}{h}\left(g\left(X_{T}^{\gamma_{t}^{h_{e_{i}}}}\right)-g\left(X_{T}^{\gamma_{t}}\right)\right) \\
& \quad-\int_{s}^{T}\left[\frac{1}{h} \widehat{\alpha}_{\gamma_{t}, \gamma_{t} h_{e_{i}}}(r)+\widehat{\beta}_{\gamma_{t}, \gamma_{t} h_{e_{i}}}(r) \Delta_{h}^{i} Y^{\gamma_{t}}(r)\right. \\
& \quad+\widehat{\delta}_{\left.\gamma_{t}, \gamma_{t}{ }_{h_{i}} \Delta_{h}^{i} Z^{\gamma_{t}}(r)\right] d r} \\
& \quad-\int_{t}^{T} \Delta_{h}^{i} Z^{\gamma_{t}}(r) d W(r) .
\end{aligned}
$$

Then the same calculus implies that

$$
\begin{gathered}
E\left[\sup _{s \in[t, T]}\left|\Delta_{h}^{i} Y^{\gamma_{t}}(s)\right|^{p}+\left.\left.\left|\int_{t}^{T}\right| \Delta_{h}^{i} Z^{\gamma_{t}}\right|^{2} d r\right|^{p / 2}\right] \\
\leq C_{2}\left(1+\left\|\gamma_{t}\right\|^{q}+|h|^{q}\right) .
\end{gathered}
$$

Consider

$$
\begin{aligned}
& \Delta_{h}^{i} Y^{\gamma_{t}}(s)-\Delta_{\bar{h}}^{i} Y^{\bar{\gamma}_{\bar{t}}}(s) \\
& =\frac{1}{h}\left(g\left(X_{T}^{\gamma_{t}^{h_{e_{i}}}}\right)-g\left(X_{T}^{\gamma_{t}}\right)\right)-\frac{1}{\bar{h}}\left(g\left(X_{T}^{\bar{\gamma}_{t}^{\bar{h}_{e_{i}}}}\right)-g\left(X_{T}^{\bar{\gamma}_{\bar{t}}}\right)\right) \\
& -\int_{s}^{T}\left(\Delta_{h}^{i} Z^{\gamma_{t}}(r)-\Delta_{\bar{h}}^{i} Z^{\bar{\gamma}_{\bar{t}}}(r)\right) d W(r) \\
& -\left\{\int _ { s } ^ { T } \left[\frac{1}{h} \widehat{\alpha}_{\gamma_{t}, \gamma_{t}^{h_{e_{i}}}}(r)-\frac{1}{\bar{h}} \widehat{\alpha}_{\bar{\gamma}_{\bar{t}}, \bar{\gamma}_{\bar{t}}}(r)\right.\right. \\
& +\widehat{\beta}_{\gamma_{t}, \gamma_{t} h_{e_{i}}}(r) \Delta_{h}^{i} Y^{\gamma_{t}}(r)-\widehat{\beta}_{\bar{\gamma}_{\bar{t}}, \bar{\gamma}_{e_{\bar{t}}}}(r) \Delta_{\bar{h}}^{i} Y^{\bar{\gamma}_{\bar{t}}}(r) \\
& \left.\left.+\widehat{\delta}_{\gamma_{t}, \gamma_{t}} \Delta_{h}^{i} Z^{\gamma_{t}}(r)-\widehat{\delta}_{\bar{\gamma}_{\bar{t}}, \bar{\gamma}_{\bar{t}}}(r) \Delta_{\bar{h}}^{i} Z^{\bar{\gamma}_{\bar{t}}}(r)\right] d r\right\} .
\end{aligned}
$$

Set

$$
\begin{aligned}
& (\widetilde{Y}(s), \widetilde{Z}(s)) \\
& \quad:=\left(\Delta_{h}^{i} Y^{\gamma_{t}}(s)-\Delta_{\bar{h}}^{i} Y^{\bar{\gamma}_{\bar{t}}}(s), \Delta_{h}^{i} Z^{\gamma_{t}}(s)-\Delta_{\bar{h}}^{i} Z^{\bar{\gamma}_{\bar{t}}}(s)\right) .
\end{aligned}
$$


Then it solves the following BSDE:

$\widetilde{Y}(s)$

$$
\begin{aligned}
= & \frac{1}{h}\left(g\left(X_{T}^{\gamma_{t}^{h_{e_{i}}}}\right)-g\left(X_{T}^{\gamma_{t}}\right)\right)-\frac{1}{\bar{h}}\left(g\left(X_{T}^{\bar{\gamma}_{t}^{\bar{h}_{t}}}\right)-g\left(X_{T}^{\bar{\gamma}_{\bar{t}}}\right)\right) \\
& -\int_{s}^{T}\left[\widehat{\beta}_{\gamma_{t} \gamma_{t} h_{e_{i}}}(r) \widetilde{Y}(r)+\widehat{\delta}_{\gamma_{t}, \gamma_{t}}{ }_{h_{t}} \widetilde{Z}(r)+\widetilde{h}(r)\right] d r \\
& -\int_{s}^{T} \widetilde{Z}(r) d W(r),
\end{aligned}
$$

where

$$
\begin{aligned}
& \widetilde{h}(r) \\
& :=\left[\widehat{\beta}_{\gamma_{t}, \gamma_{t} h_{e_{i}}}(r)-\widehat{\beta}_{\bar{\gamma}_{\bar{t}} \overline{\bar{\gamma}_{\bar{t}}} \overline{\bar{p}}_{\bar{t}}}(r)\right] \Delta_{\bar{h}}^{i} Y^{\overline{\gamma_{\bar{t}}}}(r) \\
& +\left[\widehat{\delta}_{\gamma_{t}, \gamma_{t}^{h_{e_{i}}}}(r)-\widehat{\delta}_{\bar{\gamma}_{\bar{t}} \cdot \bar{\gamma}_{\bar{t}}}(r)\right] \Delta_{\bar{h}}^{i} Z^{\bar{\gamma}_{\bar{t}}}(r) \\
& +\frac{1}{h} \widehat{\alpha} \widehat{\gamma}_{t} \gamma_{t}^{h_{e_{i}}}(r)-\frac{1}{\bar{h}} \widehat{\alpha}_{\bar{\gamma}_{t}, \bar{\gamma}_{\bar{t}}}{\overline{\bar{h}_{i}}}_{\bar{t}}(r) .
\end{aligned}
$$

Thus, under Assumptions 10, 11, similarly as in Lemma 12, we can get the last three inequalities.

Theorem 14. For each $\gamma_{t} \in \Lambda,\left\{Y_{t}^{\gamma_{t}^{z}}(s), s \in[t, T], z \in \mathbb{R}^{n}\right\}$ has a version which is a.e. of class $C^{0,2}\left([0, T] \times \mathbb{R}^{n}\right)$.

Proof. We only consider one dimensional case. Applying Lemma 12 , for each $h, \bar{h} \in \mathbb{R} \backslash\{0\}$ and $k, \bar{k} \in \mathbb{R}$,

$$
\begin{aligned}
& E\left[\sup _{u \in[t, T]}\left|Y^{\gamma_{t}^{k}}(u)-Y^{\gamma_{t}^{\bar{k}}}(u)\right|^{p}\right] \leq C_{p}\left(1+\left\|\gamma_{t}\right\|^{q}\right)|k-\bar{k}|^{p}, \\
& E\left[\left|\int_{t}^{T}\right| Z^{\gamma_{t}^{k}}(u)-\left.\left.Z^{\gamma_{t}^{\bar{k}}}\right|^{p} d u\right|^{p / 2}\right] \leq C_{2}\left(1+\left\|\gamma_{t}\right\|^{q}\right)|k-\bar{k}|^{p}, \\
& E\left[\sup _{u \in[t, T]}\left|\Delta_{h}^{i} Y^{\gamma_{t}^{k}}(u)-\Delta_{\bar{h}}^{i} Y^{\gamma_{t}^{\bar{k}}}\right|^{p}\right] \\
& \leq C_{2}\left(1+\left\|\gamma_{t}\right\|^{q}+\left\|\bar{\gamma}_{\bar{t}}\right\|^{q}+|h|^{q}+|\bar{h}|^{q}\right) \\
& \quad \times\left(|k-\bar{k}|^{p}+|h-\bar{h}|^{p}\right), \\
& E\left[\left|\int_{t}^{T}\right| \Delta_{h}^{i} Z^{\gamma_{t}^{k}}(u)-\left.\left.\Delta_{\bar{h}}^{i} Z^{\gamma_{t}^{\bar{k}}}(u)\right|^{2} d u\right|^{p / 2}\right] \\
& \leq C_{2}\left(1+\left\|\gamma_{t}\right\|^{q}+\left\|\bar{\gamma}_{\bar{t}}\right\|^{q}+|h|^{q}+|\bar{h}|^{q}\right) \\
& \quad \times\left(|k-\bar{k}|^{p}+|h-\bar{h}|^{p}\right) .
\end{aligned}
$$

By Kolmogorov's criterion, there exists a continuous derivative of $Y^{\gamma_{t}^{z}}(s)$ with respect to $z$. There also exists a meansquare derivative of $Z^{\gamma_{t}^{z}}(s)$ with respect to $z$, which is mean square continuous in $z$. We denote them by

$$
\left(D_{z} Y^{\gamma_{t}}, D_{z} Z^{\gamma_{t}}\right) \text {. }
$$

By Theorem 13 and Definition 9, $\left(D_{z} Y^{\gamma_{t}}, D_{z} Z^{\gamma_{t}}\right)$ is the solution of the following BSDE:

$$
\begin{aligned}
D_{x} Y^{\gamma_{t}}(s)= & g_{\gamma_{t}}^{\prime}\left(X_{T}^{\gamma_{t}}\right)-\int_{s}^{T} D_{x} Z^{\gamma_{t}}(r) d W(r) \\
-\int_{s}^{T}[ & h_{\gamma_{t}}^{\prime}\left(X_{r}^{\gamma_{t}}, Y^{\gamma_{t}}(r), Z^{\gamma_{t}}(r)\right) \\
& +h_{y}^{\prime}\left(X_{r}^{\gamma_{t}}, Y^{\gamma_{t}}(r), Z^{\gamma_{t}}(r)\right) D_{x} Y^{\gamma_{t}}(r) \\
& \left.+h_{z}^{\prime}\left(X_{r}^{\gamma_{t}}, Y^{\gamma_{t}}(r), Z^{\gamma_{t}}(r)\right) D_{x} Z^{\gamma_{t}}(r)\right] d r .
\end{aligned}
$$

It is easy to check that the above BSDE has a unique solution. Thus the existence of a continuous second order derivative of $Y^{\gamma_{t}^{z}}(s)$ with respect to $z$ is proved in a similar way.

Define

$$
u\left(\gamma_{t}\right):=Y^{\gamma_{t}}(t), \quad \text { for } \gamma_{t} \in \Lambda .
$$

We have the following results about $u\left(\gamma_{t}\right)$.

Lemma 15. For all $t_{1} \leq t \leq T$, one has $u\left(X_{t}^{\gamma_{t_{1}}}\right)=Y^{\gamma_{t_{1}}}(t)$.

Proof. For given $\gamma_{t_{1}}, t_{1}<t$, set $X_{t_{1}}=\gamma_{t_{1}}$. Consider the solutions of FBSDE (5) and (6) on $[t, T]$ :

$$
\begin{aligned}
X^{\gamma_{t_{1}}} & (s)=X^{\gamma_{t_{1}}}(t)+\int_{t}^{s} b\left(X_{r}^{\gamma_{t_{1}}}\right) d r+\int_{t}^{s} \sigma\left(X_{r}^{\gamma_{t_{1}}}\right) d W(r), \\
Y^{\gamma_{t_{1}}}(s) & \\
= & g\left(X_{T}^{\gamma_{t_{1}}}\right)-\int_{s}^{T} h\left(X_{r}^{\gamma_{t_{1}}}, Y^{\gamma_{t_{1}}}(r), Z^{\gamma_{t_{1}}}(r)\right) d r \\
& -\int_{s}^{T} Z^{\gamma_{t_{1}}}(r) d W(r), \quad s \in[t, T] .
\end{aligned}
$$

We need to prove $u\left(X_{t}^{\gamma_{t_{1}}}\right)=Y^{\gamma_{t_{1}}}(t)$.

For each fixed $t \in[0, T]$ and positive integer $n$, we introduce a mapping $\gamma^{n}\left(\bar{\gamma}_{s}\right): \Lambda_{s} \mapsto \Lambda_{s}$

$$
\begin{aligned}
\gamma^{n}\left(\bar{\gamma}_{s}\right)(r)= & \bar{\gamma}_{s}(r) I_{\left[0, t_{1}\right)} \\
& +\sum_{k=0}^{n-1} \bar{\gamma}_{s}\left(t_{k+1}^{n} \wedge s\right) I_{\left[t_{k}^{n} \wedge s, t_{k+1}^{n} \wedge s\right)}(r)+\bar{\gamma}_{s}(s) I_{\{s\}}(r), \\
& s \in[0, t],
\end{aligned}
$$

where $t_{k}^{n}=t_{1}+\left(k\left(t-t_{1}\right) / n\right), k=0,1, \ldots, n$,

Denote

$$
\gamma^{n}\left(X_{t}^{\gamma_{t_{1}}}\right)(r)=X^{n, \gamma_{t_{1}}}(r), \quad t_{1} \leq r \leq t .
$$


Set

$$
X_{t}^{n, N ; \gamma_{t_{1}}}:=\sum_{i=1}^{N} I_{A_{i}} x_{t}^{i}
$$

where $\left\{A_{i}\right\}_{i=1}^{N}$ is a division of $\mathscr{F}_{t}, x_{t}^{i} \in \Lambda_{t}, i=1,2, \ldots, N$. For any $i,\left(Y^{x_{t}^{i}}(s), Z^{x_{t}^{i}}(s)\right)$ is the solution of the following BSDE:

$$
\begin{aligned}
Y^{x_{t}^{i}}(s)= & g\left(X_{T}^{x_{t}^{i}}\right) \\
& -\int_{s}^{T} h\left(X_{r}^{x_{t}^{i}}, Y^{x_{t}^{i}}(r), Z^{x_{t}^{i}}(r)\right) d r \\
& -\int_{s}^{T} Z^{x_{t}^{i}}(r) d W(r), \quad s \in[t, T] .
\end{aligned}
$$

Multiplying by $I_{A_{i}}$ and adding the corresponding terms, we obtain

$$
\begin{aligned}
& \sum_{i=1}^{N} I_{A_{i}} Y^{x_{t}^{i}}(s) \\
& =g\left(\sum_{i=1}^{N} I_{A_{i}} X_{T}^{x_{t}^{i}}\right) \\
& \quad-\int_{s}^{T} h\left(\sum_{i=1}^{N} I_{A_{i}} X_{r}^{x_{t}^{i}}, \sum_{i=1}^{N} I_{A_{i}} Y^{x_{t}^{i}}(r), \sum_{i=1}^{N} I_{A_{i}} Z^{x_{t}^{i}}(r)\right) d r \\
& \quad-\int_{s}^{T} \sum_{i=1}^{N} I_{A_{i}} Z^{x_{t}^{i}}(r) d W(r), \quad s \in[t, T] .
\end{aligned}
$$

By the uniqueness and existence theorem of BSDE, we have

$$
\begin{array}{r}
Y^{X_{t}^{n, N ; \eta_{1}}}(s)=\sum_{i=1}^{N} I_{A_{i}} Y^{x_{t}^{i}}(s), \quad Z^{X_{t}^{n, N ; * t_{1}}}(s)=\sum_{i=1}^{N} I_{A_{i}} Z^{x_{t}^{i}}(s), \\
P \text {-a.s. }
\end{array}
$$

Then, by the definition of $u$, we get

$$
Y^{X_{t}^{n, N ; \gamma_{t_{1}}}}(t)=\sum_{i=1}^{N} I_{A_{i}} Y^{x_{t}^{i}}(t)=\sum_{i=1}^{N} I_{A_{i}} u\left(x_{t}^{i}\right)=u\left(X_{t}^{n, N ; \gamma_{t_{1}}}\right) .
$$

Note that

$$
\lim _{n, N \rightarrow \infty} X_{t}^{n, N ; \gamma_{t_{1}}}=X_{t}^{\gamma_{t_{1}}}, \quad P \text {-a.s. }
$$

This completes the proof.

By Theorem 13 and 14 and the definition of vertical derivative, we have the following corollary.

Corollary 16. $u\left(\gamma_{t}\right)$ is $\Lambda$-continuous and $D_{x} u\left(\gamma_{t}\right), D_{x x} u\left(\gamma_{t}\right)$ exist; moreover they are both $\Lambda$-continuous.
Proof. By Theorem 14 we know that $D_{x} u\left(\gamma_{t}\right)$ and $D_{x x} u\left(\gamma_{t}\right)$ exist. In the following, we only prove $u\left(\gamma_{t}\right)$ is $\Lambda$-continuous. The proof for the continuous property of $D_{x} u\left(\gamma_{t}\right)$ and $D_{x x} u\left(\gamma_{t}\right)$ is similar. Taking expectation on both sides of (6),

$$
u\left(\gamma_{t}\right)=E g\left(X_{T}^{\gamma_{t}}\right)-E \int_{t}^{T} h\left(X_{r}^{\gamma_{t}}, Y^{\gamma_{t}}(r), Z^{\gamma_{t}}(r)\right) d r .
$$

For $\gamma_{t}, \bar{\gamma}_{\bar{t}} \in \Lambda, \bar{t} \geq t$, we have

$$
\begin{aligned}
& \left|u\left(\gamma_{t}\right)-u\left(\bar{\gamma}_{\bar{t}}\right)\right| \\
& \leq E\left[\left|g\left(X_{T}^{\gamma_{t}}\right)-g\left(X_{T}^{\bar{\gamma}_{\bar{t}}}\right)\right|\right] \\
& +E\left[\int_{t}^{\bar{t}}\left|h\left(X_{r}^{\gamma_{t}}, Y^{\gamma_{t}}(r), Z^{\gamma_{t}}(r)\right)\right| d r\right] \\
& +E\left[\int_{\bar{t}}^{T} \mid h\left(X_{r}^{\gamma_{t}}, Y^{\gamma_{t}}(r), Z^{\gamma_{t}}(r)\right)\right. \\
& \left.\quad-h\left(X_{r}^{\overline{\gamma_{\bar{t}}}}, Y^{\overline{\gamma_{\bar{t}}}}(r), Z^{\bar{\gamma}_{\bar{t}}}(r)\right) \mid d r\right] \\
& \leq E\left[C_{1}\left(1+\left\|X_{T}^{\gamma_{t}}\right\|^{k}+\left\|X_{T}^{\bar{\gamma}_{\bar{t}}}\right\|^{k}\right)\left\|\gamma_{t}-\bar{\gamma}_{\bar{t}}\right\|\right. \\
& +3(\bar{t}-t)^{1 / 2}\left(\int _ { t } ^ { \overline { t } } \left(\left|h\left(X^{\bar{\gamma}_{\bar{t}}}(r), 0,0\right)\right|^{2}\right.\right. \\
& \left.\left.\quad+\left|C Y^{\bar{\gamma}_{\bar{t}}}(r)\right|^{2}+\left|C Y^{\bar{\gamma}_{\bar{t}}}(r)\right|^{2}\right) d r\right)^{1 / 2} \\
& \left.+C \int_{\bar{t}}^{T}\left(\left|Y^{\gamma_{t}}(r)-Y^{\bar{\gamma}_{\bar{t}}}(r)\right|+\left|Z^{\gamma_{t}}(r)-Z^{\bar{\gamma}_{\bar{t}}}(r)\right|\right) d r\right] .
\end{aligned}
$$

By Theorem 13, for some constant $C_{1}$ depending on $C, k$, and $T$,

$$
\begin{aligned}
& \left|u\left(\gamma_{t}\right)-u\left(\bar{\gamma}_{\bar{t}}\right)\right| \\
& \quad \leq C_{1}\left(1+\left\|\gamma_{t}\right\|^{k}+\left\|\bar{\gamma}_{\bar{t}}\right\|^{k}\right)\left(\left\|\gamma_{t}-\bar{\gamma}_{\bar{t}}\right\|+|t-\bar{t}|^{1 / 2}\right) .
\end{aligned}
$$

This completes the proof.

3.2. Path Regularity of Process Z. In Pardoux and Peng [2], BSDE is only state-dependent, that is, $h=h(t, \gamma(t), y, z)$ and $g=g(\gamma(T))$. Under appropriate assumptions, $Y$ and $Z$ are related in the following sense:

$$
Z^{\gamma_{t}}(s)=\nabla_{x} u\left(s, \gamma_{t}(t)+W(s)-W(t)\right), \quad P \text {-a.s. }
$$

Under the conditions $\left(\mathbf{H}_{1}\right)$ and $\left(\mathbf{H}_{2}\right)$ in [8], Peng and Wang extend this result to the path-dependent case. The corresponding BSDE is

$$
\begin{aligned}
Y^{\gamma_{t}} & (s) \\
= & g\left(W_{T}^{\gamma_{t}}\right)-\int_{s}^{T} h\left(W_{r}^{\gamma_{t}}, Y^{\gamma_{t}}(r), Z^{\gamma_{t}}(r)\right) d r \\
& -\int_{s}^{T} Z^{\gamma_{t}}(r) d W(r), \quad s \in[t, T],
\end{aligned}
$$


where

$$
W_{T}^{\gamma_{t}}=I_{s \leq t} \gamma_{t}(s)+I_{t<s \leq T}\left(\gamma_{t}(t)+W(s)-W(t)\right) .
$$

Then under some assumptions, they obtained

$$
Z^{\gamma_{t}}(s)=D_{x} u\left(W_{s}^{\gamma_{t}}\right), \quad P \text {-a.s. }
$$

In our context, we have the following theorem.

Theorem 17. Under Assumptions 1, 10, and 11, for each $\gamma_{t} \in$ $\Lambda$, the process $\left(Z^{\gamma_{t}}(s)\right)_{s \in[t, T]}$ has a continuous version with the form

$$
Z^{\gamma_{t}}(s)=\sigma\left(X_{s}^{\gamma_{t}}\right) D_{x} u\left(X_{s}^{\gamma_{t}}\right), \quad \text { for } s \in[t, T] P \text {-a.s. }
$$

To prove the above theorem, we need the following lemma essentially from Pardoux and Peng [2].

Lemma 18. Let $\gamma_{t}$ and some $\bar{t} \in[t, T]$ be given. Suppose that

$$
g(\gamma)=\varphi(\gamma(\bar{t}), \gamma(T)-\gamma(\bar{t})),
$$

where $\varphi$ is in $C_{p}^{3}\left(\mathbb{R}^{2 d} \times \mathbb{R}^{m} ; \mathbb{R}^{m}\right)$. For $b, \sigma, h$, suppose that

$$
\begin{aligned}
b\left(\gamma_{s}\right)= & b_{1}\left(s, \gamma_{s}(s)\right) I_{[0, \bar{t})}(s) \\
& +b_{2}\left(s, \gamma_{s}(s)-\gamma_{s}(\bar{t})\right) I_{[\bar{t}, T]}(s), \\
\sigma\left(\gamma_{s}\right)= & \sigma_{1}\left(s, \gamma_{s}(s)\right) I_{[0, \bar{t})}(s) \\
& +\sigma_{2}\left(s, \gamma_{s}(s)-\gamma_{s}(\bar{t})\right) I_{[\bar{t}, T]}(s), \\
h\left(\gamma_{s}, y, z\right)= & h_{1}\left(s, \gamma_{s}(s), y, z\right) I_{[0, \bar{t})}(s) \\
& +h_{2}\left(s, \gamma_{s}(s)-\gamma_{s}(\bar{t}), y, z\right) I_{[\bar{t}, T]}(s),
\end{aligned}
$$

where $b_{i}, \sigma_{i}, h_{i} \in C^{0,3}, i=1,2$. Then for each $s \in[t, T]$,

$$
Z^{\gamma_{t}}(s)=\sigma\left(X_{s}^{\gamma_{t}}\right) D_{x} u\left(X_{s}^{\gamma_{t}}\right), \quad \text { for } s \in[t, T] P \text {-a.s. }
$$

Proof. We only consider the one dimensional case.

For $s \in[\bar{t}, T]$, the FBSDE (5), (6) can be rewritten as

$$
\begin{aligned}
X^{\gamma_{s}} & (u) \\
\quad & \gamma_{s}(s)+\int_{s}^{u} b_{2}\left(r, X^{\gamma_{s}}(s)-\gamma_{s}(\bar{t})\right) d r \\
& \quad+\int_{s}^{u} \sigma_{2}\left(r, X^{\gamma_{s}}(s)-\gamma_{s}(\bar{t})\right) d W(r), \\
Y^{\gamma_{s}}(u) & \\
= & \varphi\left(\gamma_{s}(\bar{t}), X^{\gamma_{s}}(T)-\gamma_{s}(\bar{t})\right)-\int_{u}^{T} Z^{\gamma_{s}}(r) d W(r) \\
& -\int_{u}^{T} h_{2}\left(r, \gamma_{s}(\bar{t}), X^{\gamma_{s}}(r)-\gamma_{s}(\bar{t}), Y^{\gamma_{s}}(r), Z^{\gamma_{s}}(r)\right) d r,
\end{aligned}
$$

For $s \in[t, \bar{t}]$,

$$
\begin{aligned}
X^{\gamma_{s}}(u)= & \gamma_{s}(s)+\int_{s}^{u} b_{1}\left(r, X^{\gamma_{s}}(s)\right) d r \\
& +\int_{s}^{u} \sigma_{1}\left(r, X^{\gamma_{s}}(s)\right) d W(r), \\
& u \in[s, \bar{t}], \\
X^{\gamma_{s}}(u)= & X^{\gamma_{s}}(\bar{t})+\int_{\bar{t}}^{u} b_{2}\left(r, X^{\gamma_{s}}(s)-X^{\gamma_{s}}(\bar{t})\right) d r \\
& +\int_{s}^{u} \sigma_{2}\left(r, X^{\gamma_{s}}(s)-X^{\gamma_{s}}(\bar{t})\right) d W(r), \\
Y^{\gamma_{s}}(u)= & \varphi\left(X^{\gamma_{s}}(\bar{t}), X^{\gamma_{s}}(T)-X^{\gamma_{s}}(\bar{t})\right) \\
& -\int_{u}^{T} Z^{\gamma_{s}}(r) d W(r) \\
& -\int_{u}^{T} h_{2}\left(r, X^{\gamma_{s}}(\bar{t}), X^{\gamma_{s}}(r)\right. \\
& \left.-X^{\gamma_{s}}(\bar{t}), Y^{\gamma_{s}}(r), Z^{\gamma_{s}}(r)\right) d r, \\
& -\int_{u}^{\bar{t}} Z^{\gamma_{s}}(r) d W(r), \quad u \in[s, \bar{t}] . \\
& Y^{\gamma_{s}}(u) \\
= & Y^{\gamma_{s}}(\bar{t})-\int_{u}^{\bar{t}} h_{1}\left(r, X^{\gamma_{s}}(\bar{t}), Y^{\gamma_{s}}(r), Z^{\gamma_{s}}(r)\right) d r
\end{aligned}
$$

Now consider the following system of quasilinear parabolic differential equations, which is defined on $[\bar{t}, T] \times \mathbb{R}^{2}$ and parameterized by $x \in \mathbb{R}$ :

$$
\begin{gathered}
\partial_{s} u_{2}(s, x, y)+\mathscr{L} u_{2}(s, x, y) \\
=h_{2}\left(s, x, y, u_{2}(s, x, y), \partial_{y} u_{2}(s, x, y) \sigma\left(r_{s}\right)\right), \\
u_{2}(T, x, y)=\varphi(x, y),
\end{gathered}
$$

where $\mathscr{L}=(1 / 2) \sigma^{2}\left(\partial^{2} / \partial_{x x}\right)+b\left(\partial / \partial_{x}\right)$. The other one is defined on $[t, \bar{t}] \times \mathbb{R}$ :

$$
\begin{gathered}
\partial_{s} u_{1}(s, x)+\mathscr{L} u_{1}(s, x) \\
=h_{1}\left(r, x, u_{1}(s, x), \partial_{y} u_{1}(s, x) \sigma(r)\right), \\
u_{1}(\bar{t}, x)=u_{2}(\bar{t}, x, 0),
\end{gathered}
$$

where $\mathscr{L}=(1 / 2) \sigma^{2}\left(\partial^{2} / \partial_{x x}\right)+b\left(\partial / \partial_{x}\right)$. By Corollary 16 and Theorems 3.1, 3.2 in Paroux-Peng [2], we have $u_{2} \in$ $C^{1,2}\left([\bar{t}, T] \times \mathbb{R}^{2} ; \mathbb{R}\right), u_{1} \in C^{1,2}([t, \bar{t}] \times \mathbb{R} ; \mathbb{R})$, and

$$
\begin{aligned}
u\left(\gamma_{s}\right)= & u_{1}\left(s, \gamma_{s}(s)\right) I_{[t, \bar{t})}(s) \\
& +u_{2}\left(s, \gamma_{s}(\bar{t}), \gamma_{s}(s)-\gamma_{s}(\bar{t})\right) I_{[\bar{t}, T]}(s) .
\end{aligned}
$$


Then we obtain

$$
\begin{aligned}
& Y^{\gamma_{t}}(s)=u_{1}\left(s, X^{\gamma_{t}}(s)\right), \quad t \leq s<\bar{t}, \\
& Y^{\gamma_{t}}(s)=u_{2}\left(s, X^{\gamma_{t}}(\bar{t}), X^{\gamma_{t}}(s)-X^{\gamma_{t}}(\bar{t})\right), \quad \bar{t} \leq s \leq T, \\
& Z^{\gamma_{t}}(s)=\partial_{x} u_{1}\left(s, X^{\gamma_{t}}(s)\right) \sigma_{1}\left(X_{s}^{\gamma_{t}}\right), \quad t \leq s<\bar{t}, \\
& Z^{\gamma_{t}}(s)=\partial_{x} u_{2}\left(s, X^{\gamma_{t}}(\bar{t}), X^{\gamma_{t}}(s)-X^{\gamma_{t}}(\bar{t})\right) \sigma_{2}\left(X_{s}^{\gamma_{t}}\right), \\
& \bar{t} \leq s \leq T .
\end{aligned}
$$

Finally, for each $s \in[t, T]$,

$$
Z^{\gamma_{t}}(s)=\sigma\left(X_{s}^{\gamma_{t}}\right) D_{x} u\left(X_{s}^{\gamma_{t}}\right), \quad P \text {-a.s. }
$$

In particular,

$$
Z^{\gamma_{t}}(t)=\sigma\left(\gamma_{t}\right) D_{x} u\left(\gamma_{t}\right), \quad \gamma_{t} \in \Lambda
$$

This completes the proof.

Now we give the proof of Theorem 17.

Proof. For each fixed $t \in[0, T]$ and positive integer $n$, we introduce a mapping $\gamma^{n}\left(\bar{\gamma}_{s}\right): \Lambda_{s} \mapsto \Lambda_{s}$

$$
\begin{aligned}
\gamma^{n}\left(\bar{\gamma}_{s}\right)(r) & \\
= & \bar{\gamma}_{s}(r) I_{[0, t)}+\sum_{k=0}^{n-1} \bar{\gamma}_{s}\left(t_{k+1}^{n} \wedge s\right) I_{\left[t_{k}^{n} \wedge s, t_{k+1}^{n} \wedge s\right)}(r) \\
& +\bar{\gamma}_{s}(s) I_{\{s\}}(r), \quad s \in[0, T],
\end{aligned}
$$

where $t_{k}^{n}=t+(k(T-t) / n), k=0,1, \ldots, n$

$$
g^{n}(\bar{\gamma}):=g\left(\gamma^{n}(\bar{\gamma})\right), \quad h^{n}\left(\bar{\gamma}_{s}, y, z\right):=h\left(\gamma^{n}\left(\bar{\gamma}_{s}\right), y, z\right) .
$$

For each $n$, there exist some functions $\varphi_{n}$ defined on $\Lambda_{t} \times$ $\mathbb{R}^{n \times d}$ and $\psi_{n}, \phi_{n}, \widetilde{\phi}_{n}$ defined on $[t, T] \times \Lambda_{t} \times \mathbb{R}^{n \times d} \times \mathbb{R}^{m} \times \mathbb{R}^{m \times d}$ such that

$$
\begin{aligned}
& g^{n}(\bar{\gamma}) \\
& :=\varphi_{n}\left(\bar{\gamma}_{t}, \bar{\gamma}\left(t_{1}^{n}\right)-\bar{\gamma}(t), \ldots, \bar{\gamma}\left(t_{n}^{n}\right)-\bar{\gamma}\left(t_{n-1}^{n}\right)\right), \\
& b^{n}\left(\bar{\gamma}_{s}\right) \\
& \quad:=\phi_{n}\left(s, \bar{\gamma}_{t}, \bar{\gamma}_{s}\left(t_{1}^{n} \wedge s\right)-\bar{\gamma}_{s}(t), \ldots, \bar{\gamma}_{s}\left(t_{n}^{n} \wedge s\right)\right. \\
& \left.\quad-\bar{\gamma}_{s}\left(t_{n-1}^{n} \wedge s\right)\right), \\
& \left.\sigma^{n}\left(\bar{\gamma}_{s}\right) \quad-\bar{\gamma}_{s}\left(t_{n-1}^{n} \wedge s\right)\right), \\
& h^{n}\left(\bar{\gamma}_{s}, y, z\right) \quad \bar{\gamma}_{t}, \bar{\gamma}_{s}\left(t_{1}^{n} \wedge s\right)-\bar{\gamma}_{s}(t), \ldots, \bar{\gamma}_{s}\left(t_{n}^{n} \wedge s\right) \\
& :=\psi_{n}\left(s, \bar{\gamma}_{t}, \bar{\gamma}_{s}\left(t_{1}^{n} \wedge s\right)-\bar{\gamma}_{s}(t), \ldots, \bar{\gamma}_{s}\left(t_{n}^{n} \wedge s\right)\right. \\
& \left.\quad-\bar{\gamma}_{s}\left(t_{n-1}^{n} \wedge s\right), y, z\right) .
\end{aligned}
$$

Indeed, if we set

$$
\begin{gathered}
\bar{\varphi}_{n}\left(\bar{\gamma}_{t}, x_{1}, \ldots, x_{n}\right) \\
:=g\left(\left(\bar{\gamma}_{t}(s) I_{[0, t)}(s)\right.\right. \\
\left.\left.+\sum_{k=1}^{n} x_{k} I_{\left[t_{k-1}^{n}, t_{k}^{n}\right)}(s)+x_{n} I_{\{T\}}(s)\right)_{0 \leq s \leq T}\right), \\
\varphi_{n}\left(\bar{\gamma}_{t}, x_{1}, \ldots, x_{n}\right) \\
:=\bar{\varphi}_{n}\left(\bar{\gamma}_{t}, \bar{\gamma}_{t}+x_{1}, \bar{\gamma}_{t}(t)+x_{1}\right. \\
\left.+x_{2}, \ldots, \bar{\gamma}_{t}(t)+\sum_{i=1}^{n} x_{i}\right),
\end{gathered}
$$

then by Assumptions 1, 10, and 11, we obtain that, for each fixed $\bar{\gamma}_{t}, \varphi_{n}\left(\bar{\gamma}_{t}, x_{1}, \ldots, x_{n}\right)$ is a $C_{p}^{3}$-function of $x_{1}, \ldots, x_{n}$. In particular, for each $\bar{\gamma} \in \Lambda$,

$$
\begin{aligned}
\partial_{x_{i}} \varphi_{n} & \left(\bar{\gamma}_{t}, \bar{\gamma}\left(t_{1}^{n}\right)-\bar{\gamma}(t), \ldots, \bar{\gamma}\left(t_{n}^{n}\right)-\bar{\gamma}\left(t_{n-1}^{n}\right)\right) \\
& =g_{\gamma_{t_{i-1}^{n}}^{\prime}}^{\prime}\left(\gamma^{n}(\bar{\gamma})\right) .
\end{aligned}
$$

For any $\bar{t} \geq t, \bar{\gamma}_{\bar{t}} \in \Lambda_{\bar{t}}$, we consider the following FBSDE:

$$
\begin{aligned}
X^{n, \bar{\gamma}_{\bar{t}}}(s) & \\
= & \bar{\gamma}_{\bar{t}}(\bar{t})+\int_{\bar{t}}^{s} b^{n}\left(X_{r}^{n, \bar{\gamma}_{\bar{t}}}\right) d r+\int_{\bar{t}}^{s} \sigma^{n}\left(X_{r}^{n, \bar{\gamma}_{\bar{t}}}\right) d W(r), \\
Y^{n, \bar{\gamma}_{\bar{t}}}(s) & \\
= & g^{n}\left(X_{T}^{n, \bar{\gamma}_{\bar{t}}}\right)-\int_{s}^{T} h^{n}\left(X_{r}^{n, \bar{\gamma}_{\bar{t}}}, Y^{n, \bar{\gamma}_{\bar{t}}}(r), Z^{n, \bar{\gamma}_{\bar{t}}}(r)\right) d r \\
& \quad-\int_{s}^{T} Z^{n, \bar{\gamma}_{\bar{t}}}(r) d W(r), \quad \bar{t} \leq s .
\end{aligned}
$$

We denote

$$
u^{n}\left(\bar{\gamma}_{\bar{t}}\right):=Y^{n \bar{\gamma}_{\bar{t}}}(t), \quad \bar{\gamma}_{\bar{t}} \in \Lambda
$$

Following the argument as in Lemma 18, for each $s \in[t, T]$, we have

$$
Z^{n, \bar{\gamma}_{\bar{t}}}(s)=\sigma^{n}\left(X_{s}^{n, \bar{\gamma}_{\bar{t}}}\right) D_{x} u^{n}\left(\bar{\gamma}_{\bar{t}}\right), \quad P \text {-a.s. }
$$


By the definition of $g^{n}, b^{n}, \sigma^{n}, h^{n}$ and (82) we have

$$
\begin{gathered}
\lim _{n} X_{T}^{n, \bar{\gamma}_{\bar{t}}}=X_{T}^{\bar{\gamma}_{\bar{t}}}, \quad P \text {-a.s, } \\
\lim _{n}\left(Y^{n, \bar{\gamma}_{\bar{t}}}(s), Z^{n, \bar{\gamma}_{\bar{t}}}(s)\right)=\left(Y^{\bar{\gamma}_{\bar{t}}}(s), Z^{\bar{\gamma}_{\bar{t}}}(s)\right), \\
\text { a.e. } s \in[t, T], P \text {-a.s, } \\
\lim _{n} u^{n}\left(\bar{\gamma}_{\bar{t}}\right)=u\left(\bar{\gamma}_{\bar{t}}\right), \quad \lim _{n} D_{x} u^{n}\left(\bar{\gamma}_{\bar{t}}\right)=D_{x} u\left(\bar{\gamma}_{\bar{t}}\right), \\
\lim _{n} D_{x x} u^{n}\left(\bar{\gamma}_{\bar{t}}\right)=D_{x x} u\left(\bar{\gamma}_{\bar{t}}\right), \\
\left.\left.=\left(X_{s}^{n, \bar{\gamma}_{t}}\right), D_{x} u^{n}\left(X_{s}^{n, \bar{\gamma}_{t}}\right), D_{x x} u^{n}\left(X_{s}^{n, \bar{\gamma}_{t}}\right)\right), D_{x} u\left(X_{s}^{\bar{\gamma}_{t}}\right), D_{x x} u\left(X_{s}^{\bar{\gamma}_{t}}\right)\right),
\end{gathered}
$$

a.e. $s \in[t, T], P$-a.s.

Therefore

$Z^{\gamma_{t}}(s)=\sigma\left(X_{s}^{\bar{\gamma}_{t}}\right) D_{x} u\left(X_{s}^{\bar{\gamma}_{t}}\right), \quad$ a.e. $s \in[t, T], P$-a.s.

This completes the proof.

\section{The Related Parabolic \\ Path-Dependent PDEs}

In this section, we relate FBSDE (5), (6) to the following pathdependent partial differential equation:

$$
\begin{gathered}
D_{t} u\left(\gamma_{t}\right)+\mathscr{L} u\left(\gamma_{t}\right)-h\left(\gamma_{t}, u\left(\gamma_{t}\right), \sigma\left(\gamma_{t}\right) D_{x} u\left(\gamma_{t}\right)\right)=0, \\
u\left(\gamma_{T}\right)=g\left(\gamma_{T}\right), \quad \gamma_{T} \in \Lambda^{n},
\end{gathered}
$$

where

$$
\mathscr{L} u=\frac{1}{2} \operatorname{tr}\left[\left(\sigma \sigma^{T}\right) D_{x x} u\right]+\left\langle b, D_{x} u\right\rangle .
$$

Theorem 19. Suppose that Assumptions 1, 10, and 11 are fulfilled, and if $u \in \mathbb{C}^{1.2}(\Lambda)$, and that $u$ is the solution of (88), $u$ is uniformly Lipschitz continuous, and bounded by $C\left(1+\left\|\gamma_{t}\right\|\right)$, then the solution is unique, and for any $\gamma_{t} \in \Lambda, u\left(\gamma_{t}\right)$ is determined by (5) and (6).

Proof. By the assumptions of this theorem, we know that $b\left(\gamma_{t}\right)$ and $\sigma\left(\gamma_{t}\right)$ are uniformly Lipschtiz continuous and the following SDE has a uniqueness solution:

$$
\begin{gathered}
d X^{\gamma_{t}}(s)=b\left(X_{s}^{\gamma_{t}}\right) d s+\sigma\left(X_{s}^{\gamma_{t}}\right) d W(s), \\
X_{t}=\gamma_{t}, \quad s \in[t, T] .
\end{gathered}
$$

Set $Y(s)=u\left(X_{s}^{\gamma_{t}}\right), t \leq s \leq T$. Applying Itô's formula to $Y(s)=$ $u\left(X_{s}^{\gamma_{t}}\right)$, we have

$$
\begin{gathered}
d Y(s) \\
=-h\left(X_{s}^{\gamma_{t}}, Y(s), Z(s)\right) d r-\sigma\left(X_{s}^{\gamma_{t}}\right) D_{x} u\left(X_{s}^{\gamma_{t}}\right) d W(s) . \\
Y(T)=g\left(X_{T}^{\gamma_{t}}\right) \quad s \in[t, T] .
\end{gathered}
$$

Then by the uniqueness and existence theorem of the functional FBSDE, we obtain the result.

Now we prove the converse to the above result.

Theorem 20. Under Assumptions 1, 10, and 11, the function $u\left(\gamma_{t}\right)=Y^{\gamma_{t}}(t)$ is the unique $\mathbb{C}^{1,2}(\Lambda)$-solution of the pathdependent PDE (88).

Proof. We only study the one dimensional case. $u \in \mathbb{C}^{0,2}(\Lambda)$ follows from Corollary 16. Let $\delta>0$ satisfying $t+\delta \leq T$. By Lemma 15 we can get

$$
u\left(X_{t+\delta}^{\gamma_{t}}\right)=Y^{\gamma_{t}}(t+\delta)
$$

Hence

$$
\begin{aligned}
& u\left(\gamma_{t, t+\delta}\right)-u\left(\gamma_{t}\right) \\
& \quad=u\left(\gamma_{t, t+\delta}\right)-u\left(X_{t+\delta}^{\gamma_{t}}\right)+u\left(X_{t+\delta}^{\gamma_{t}}\right)-u\left(\gamma_{t}\right),
\end{aligned}
$$

By the proof of Theorem 17, we obtain

$$
\begin{aligned}
& u\left(\gamma_{t, t+\delta}\right)-u\left(\gamma_{t}\right) \\
& =\lim _{n \rightarrow \infty}\left[u^{n}\left(\gamma_{t, t+\delta}\right)-u^{n}\left(X_{t+\delta}^{\gamma_{t}}\right)\right] \\
& \quad+\int_{t}^{t+\delta} h\left(X_{s}^{\gamma_{t}}, Y^{\gamma_{t}}(s), Z^{\gamma_{t}}(s)\right) d s \\
& \quad+\int_{t}^{t+\delta} Z^{\gamma_{t}}(s) d W(s) .
\end{aligned}
$$

By Lemma 3.1 and Theorem 3.2 of Pardoux and Peng [2] and Theorem 4.4 of Peng and Wang [8], we deduce that

$$
\begin{aligned}
& u^{n}\left(\gamma_{t, t+\delta}\right)-u^{n}\left(X_{t+\delta}^{\gamma_{t}}\right) \\
& =\int_{t}^{t+\delta} D_{s} u^{n}\left(\gamma_{t, s}\right) d s-\int_{t}^{t+\delta} D_{s} u^{n}\left(X_{s}^{\gamma_{t}}\right) d s \\
& \quad-\int_{t}^{t+\delta} D_{x} u^{n}\left(X_{s}^{\gamma_{t}}\right) d X^{n, \gamma_{t}}(s) \\
& \quad-\frac{1}{2} \int_{t}^{t+\delta} D_{x x} u^{n}\left(X_{s}^{\gamma_{t}}\right) d\left\langle X^{n, \gamma_{t}}\right\rangle(s) .
\end{aligned}
$$

Thus by (86) and the dominated convergence theorem, we have

$$
\begin{aligned}
& u\left(\gamma_{t, t+\delta}\right)-u\left(\gamma_{t}\right) \\
& =-\int_{t}^{t+\delta} D_{x} u\left(X_{s}^{\gamma_{t}}\right) d X^{\gamma_{t}}(s) \\
& \quad-\frac{1}{2} \int_{t}^{t+\delta} D_{x x} u\left(X_{s}^{\gamma_{t}}\right) d\left\langle X^{\gamma_{t}}\right\rangle(s) \\
& +\int_{t}^{t+\delta} h\left(X_{s}^{\gamma_{t}}, Y^{\gamma_{t}}(s), Z^{\gamma_{t}}(s)\right) d s \\
& +\int_{t}^{t+\delta} Z^{\gamma_{t}}(s) d W(s)+\lim _{n \rightarrow \infty} C^{n}
\end{aligned}
$$


where

$$
C^{n}=\int_{t}^{t+\delta} D_{s} u^{n}\left(\gamma_{t, s}\right) d s-\int_{t}^{t+\delta} D_{s} u^{n}\left(X_{s}^{\gamma_{t}}\right) d s .
$$

Note that $u^{n}\left(\gamma_{t}\right) \in \mathbb{C}_{l, l i p}^{0,2}(\Lambda)$. By Lemma 12 , we have

$$
\begin{array}{r}
\left|D_{s} u^{n}\left(\gamma_{t, s}\right)-D_{s} u^{n}\left(X_{s}^{\gamma_{t}}\right)\right| \leq c\left\|\gamma_{t}-X_{s}^{\gamma_{t}}\right\|, \\
\text { a.e. } s \in[t, T], P \text {-a.s }
\end{array}
$$

for some constant $c$ depending on $C, T, \gamma_{t}$, and $k$. Hence

$$
\left|C^{n}\right| \leq c \delta \sup _{s \in[t, t+\delta]}\left|X^{\gamma_{t}}(s)-\gamma_{t}(t)\right|, \quad P \text {-a.s. }
$$

Taking expectation on both sides of (96), we have

$$
\begin{aligned}
\lim _{\delta \rightarrow 0} & \frac{u\left(\gamma_{t, t+\delta}\right)-u\left(\gamma_{t}\right)}{\delta} \\
& =-\mathscr{L} u\left(\gamma_{t}\right)+h\left(\gamma_{t}, u\left(\gamma_{t}\right), D_{x} u\left(\gamma_{t}\right) \sigma\left(\gamma_{t}\right)\right) .
\end{aligned}
$$

Thus $u\left(\gamma_{t}\right)$ belongs to $\mathbb{C}^{1,2}(\Lambda)$ and satisfies (88).

\section{Acknowledgments}

This work was supported by National Natural Science Foundation of China (no. 11171187 and no. 11221061); by the Programme of Introducing Talents of Discipline to Universities of China (no. B12023); and by Program for New Century Excellent Talents in University of China.

\section{References}

[1] S. G. Peng, "Probabilistic interpretation for systems of quasilinear parabolic partial differential equations," Stochastics and Stochastics Reports, vol. 37, no. 1-2, pp. 61-74, 1991.

[2] É. Pardoux and S. Peng, "Backward stochastic differential equations and quasilinear parabolic partial differential equations," in Stochastic Partial Diferential Equation and Their Applications, B. L. Rozuvskii and R. B. Soeers, Eds., vol. 176 of Lecture Notes in Control and Inform. Sci., pp. 200-217, Springer, Berlin, Germany, 1992.

[3] E. Pardoux and S. Tang, "Forward-backward stochastic differential equations and quasilinear parabolic PDEs," Probability Theory and Related Fields, vol. 114, no. 2, pp. 123-150, 1999.

[4] B. Dupire, "Functional Ito calculus," Portfolio Research Paper, Bloomberg, New York, NY, 2009.

[5] R. Cont and D. A. Fourni, "Functional Ito calculus and stochastic intergral representation of martingles," Annals of Probability, vol. 41, no. 1, pp. 109-133, 2010.

[6] R. Cont and D. Fournie, "A functional extension of the Ito formula," Comptes Rendus Mathématique, vol. 348, no. 1-2, pp. 57-61, 2010.

[7] R. Cont and D.-A. Fournié, "Change of variable formulas for non-anticipative functionals on path space," Journal of Functional Analysis, vol. 259, no. 4, pp. 1043-1072, 2010.

[8] S. Peng and F. Wang, "BSDE, path-dependent PDE and nonlinear Feynman-Kac formula ," http://arxiv.org/abs/1108.4317.

[9] S. Peng, "Note on viscosity solution of path-dependent PDE and G-martingales ," http://arxiv.org/abs/1106.1144 .
[10] I. Ekren, C. Keller, N. Touzi, and J. Zhang, "On viscosity solutions of path dependent PDEs," http://arxiv.org/abs/1109.5971.

[11] A. Cosso, "Viscosity solutions of path-dependent PDEs and non-Markovian forward-backward stochastic systems," http:// arxiv.org/abs/1202.2502.

[12] R. S. Lipster and A. N. Shiryaev, Statistics of Random Processes I, Springer, Berlin, Germany, 1978.

[13] E. Pardoux and S. Peng, "Adapted solution of a backward stochastic differential equation," Systems and Control Letters, vol. 14, no. 1, pp. 55-61, 1990.

[14] J. Ma and J. Yong, Forward-Backward Stochastic Differential Equations and Their Applications, vol. 1702 of Lecture Notes in Mathematics, Springer, Berlin, Germany, 1999. 


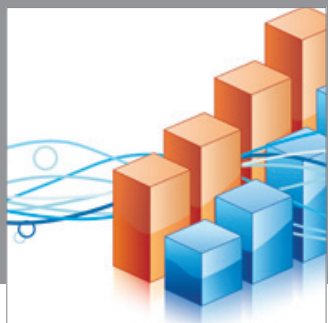

Advances in

Operations Research

mansans

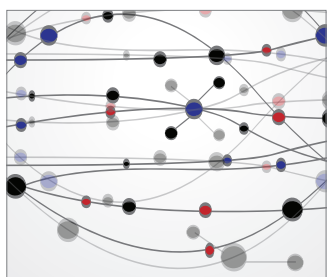

The Scientific World Journal
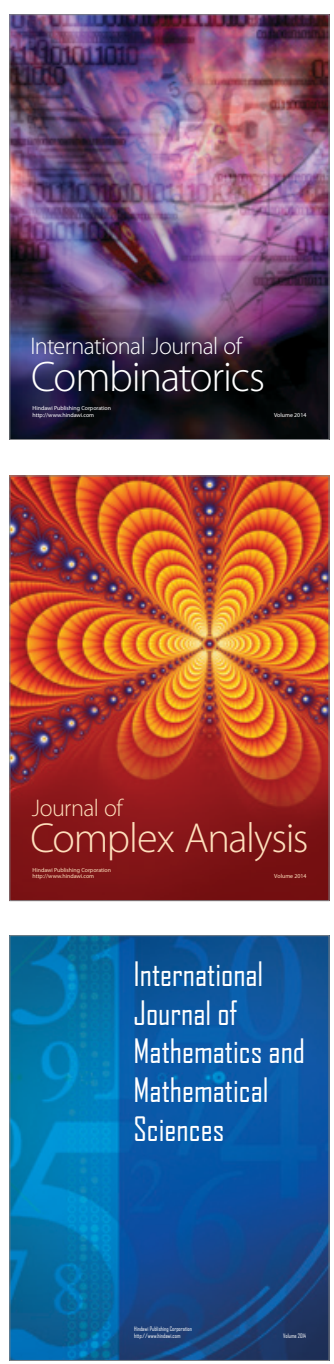
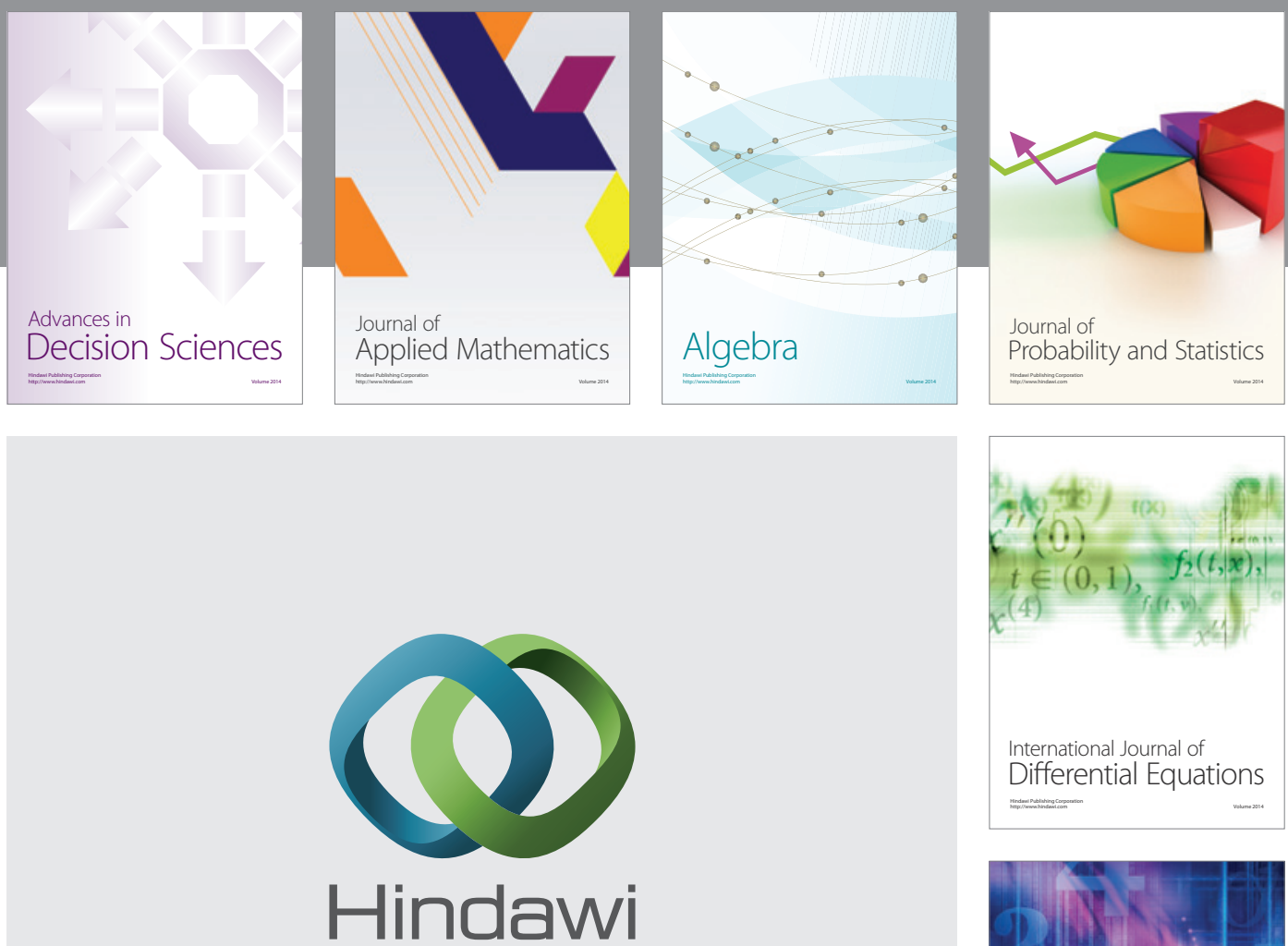

Submit your manuscripts at http://www.hindawi.com
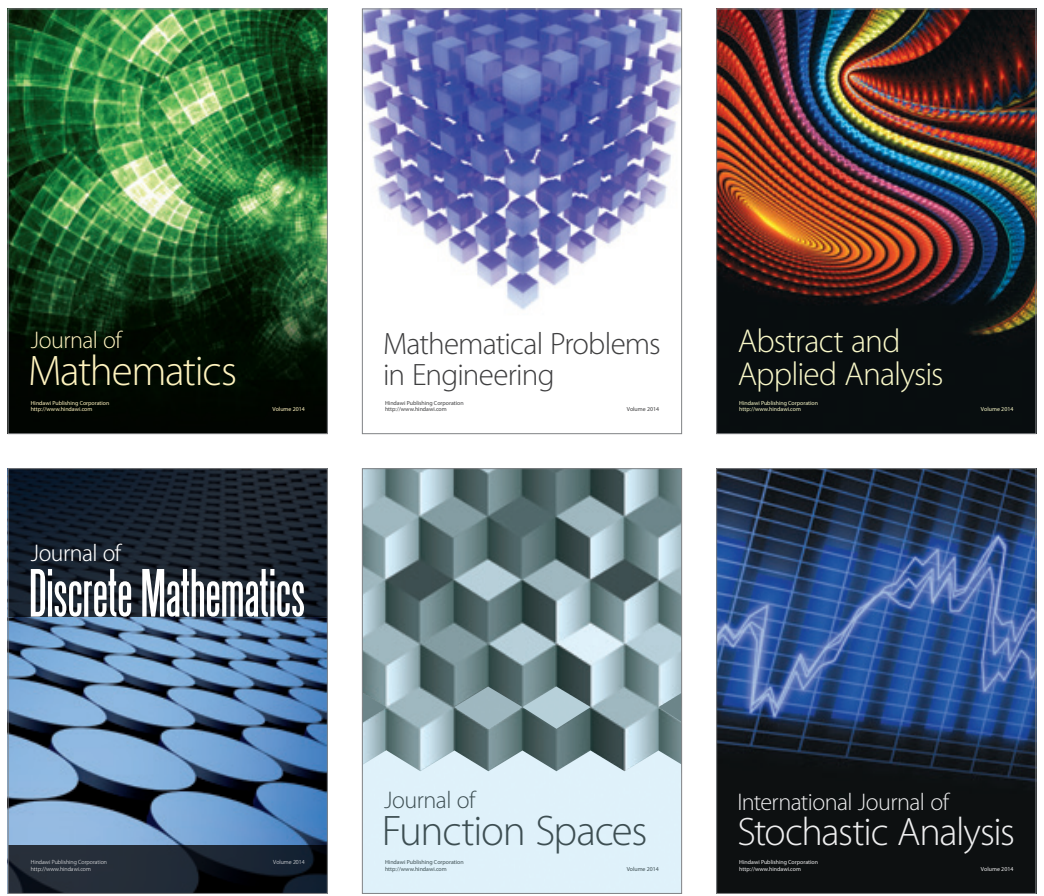

Journal of

Function Spaces

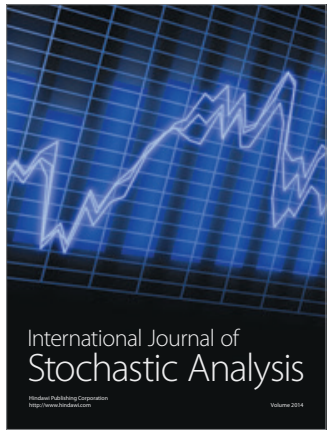

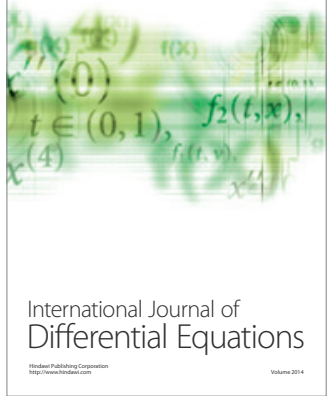
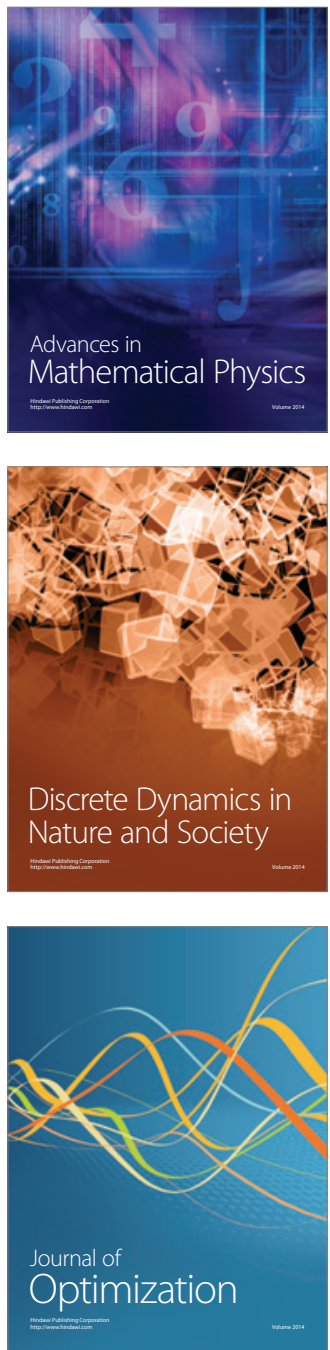